III.

\title{
Bericht über die holländische ophthalmologische Litteratur des Jahres 1903.
}

\author{
(2. Semester.)
}

Von

\author{
I)r. G. J. SCHOUTE und Prof. I)r. W. KOSTER G\%n. \\ in A msterdam. \\ in Leiden.
}

M. R. = Medische Revue.

M. W. = Medisch Weekblad.

N. T. G. = Nederlandsch Tijdschrift voor Geneeskunde.

Die mit versehenen Nummern werden weiter unten referiert.

1. Amstel, P. J. de Bruine Ploos van, Ophthalmia neonatorum. M. W. S. 189.

2. "Bijlsma, K., Onze zintuigen (Unsere Sinnesorgane). Amsterdam, F. van Rossen.

3. Blaau M. W. S. 161 .

4. "Bouvin, M. J., Pemphigus conjunctivae. N. T. G. Il. S. 969.

5. Duuring, C. P., Skiaskoop. N. T. G. II. S. 976.

6. Dyckmeester, H., Een geval van gepigmenteerde papilla nervi optici (Ein Fall von pigmentierter Sehnervenpipille). N. T. G. II. S. 82.

7. "Elias, J. PL., De pupilreflexen (Die Pupillenreflexe). M. W. S. 334, 361,387 und 449.

8. Waber, E., Papilla conica. N. T. G. II. S. 960.

9. Geuns, J. R. van, Vena optico-ciliaris. N. T. G. II. S. 950.

10. Derselbe, Arterielus in het glasvocht (Arterienschlinge im Glaskörper). N. T. G. II. S. 951.

11. "Haaften, A. W. van, Orer de beteckenis der staphylococcen voor het ontstaan der ophthalmia scrophulosa s. eczematosa (Die Rolle der Staphylokokken in der Erzeugung der Conjunctivitis scrophulosa s. eczematosa). lnaug. Diss. Amsterdam.

12. "Has, H. K. de, Lichtprikkels en retinastroomen in hun quantitatief rerband (Die quantitative Beziehung 2 ischen Lichtreizen und Netzhautströmen). Inaug.-Diss. Leiden.

13. *Hazewinkel, C., Siderosis bulbi. N. T. G. II. S. 964.

14. Derselbe, Een familie met epicanthus en ptosis (Eine Familie mit Epicanthus und Ptosisj. N. T. G. II. S. 9 j3.

15. Hildesheim, E., De blik onzer oogen (Der Blick unsrer Augen). Populäre Angesichtskunde. Groningen, van der Klei.

16. "Lechner, C. S., Aangeboren woordblindheid (Angeborene Wortblindheit). N. T. G. II. S. 235 .

17. Mulder, M. E., Inrichting voor ooglijders te Groningen (Augenklinik in Groningen). Bericht für 1902.

18. N. N., Spart uwe oogen (Schont die Augen). Rotterdan, C. Moser.

19. Nicolai, C., J. L. A. Kremer als oogarts. N. T. G. II. S. 953.

20. Noyon, J., Carcinoma parotidis en de gevolgen daarvan (Carcinoma parotidis und dessen Folgen). N. T. G. II. S. 965. 
21. "Peeters, Bestalat er glauconal door gemoedsatandoening veroorzalit:" (Gibt es ein Glankom, rerursacht durch Gemütserregung!. M. W. S. 394.

22. *Pinkh of, H., Ge\%onde oogen (Gesunde Augen). Amsterd., F. ran Rosien.

23. Pulle, A. W., Het onderzoek naar het kleuronderscheidingsiermogen bij de Kon. Ned. Marine (Die Untersuchung des Furbensinnes bei der Königl. Niederländ. Marine). (Komisch-polemischen Inhalts.) W. W. S. 196.

24. "Rochat, G. F., Sympathische Ophthalmie. N. T. G. II. S. 969.

25. *Ruys, J. A., Acute dubbelzijdige abducensparalyse (Akute, doppelseitige Abducenslähmung). N. T. G. II. S. 1405.

26. "Salomonson, J.K. A. Wertheim, Ulcus rodens en X-stralen (Die Behnadlung des Ulcus rodens mittelst X-Strahlen). N. I. G. II, S. 64\%.

27. Schoute, G. J., Een geral ran cinchonine-intoxicatie (Ein liall ron Cinchonin-Intoxikattion). N. T. G. Il. S. 25. (In dieser \%eitschrift erschienen.)

28. "Derselbe, Enophthalmus. M. R. S. 481.

29. Snellen, H. Sr., Ooglueelkundige verslagen en bijßladen No. 44 (Berichte über 190\% aus verschiedenen hollïndischen Augenkliniken. Utrecht, Boekhoven.

30. "Sturk, W. A., Hoe herkent men oogriekten bij kiuderen (Wie erkennt man Augenkrankheiten bei Kindern?). A:nhem, Gouda Quint.

31. "Straub, M., Gezichts\%akte door niet-gebruik (Sehschwäche durch Nicht.Gel,rauch). N. T. G. II. S. 590.

32. "Derselbe, Het mes van Taylor bij oogoperatios (Das Taylorsche Messer für Augenoperationen). N. T. G. II. S. 634 .

33. Vries, W. M. de, Een geval ran atngeboren oogmisvorming (liin liall ron angeborener Missbildung des Auges). N. T. G. Il. S. 38\%.

I) Vries (33) berichtet iiber ein Auge: welches enukleiert worden war, weil ein sich vergrösserncler weisser liegel auf der linsenhinterfläche fïr Gliom imponiert hatte; aus diesem legel rog ein feincr. Faden zum $N$. opticus (s. vorigen Bericht). J)as Auge hatte unten medial ein Coloboma iridis, ron dessen unterer Spitze ein bild incteilter Fortsat\% nach der Linsenhinterfliche \%og; auch ron dem Ziliarkürper zogen drei solche fortsätze nach der nänlichen Stelle. Sie waren endwegs von einem Net\%antausläufer belileidet, sodass die Ora serrata sehr unregelmässig gebildet war. Die mikroskopische Ǔntersuchung ergab, dass einer dieser Stränge aus einer Duplikatur des inneren Blattes des Augenbechers bestand; ein anderer aus einer bindegewebigen Grundmasse mit doppelter Epithelbekleidung durch beide IBlitter des Augenbechers, also ein rerlïngerter Processus ciliaris. Die Stringe. welche aus dem Colobom kamen, stellten die unmittelbare Fortset\%ung des Corpus ciliare vor: hier also fanden sich die beiden Netzhautblitter auf der Hinterseite der Stränge. (Diese Besonderheiten, sowie auch die übrigen Anomalien, welche im rorigen Berichte nur gestreift worden sind, sind dentsch ausführlich beschrieben in der anatomischen Zeitschrift "Petrus Camper". II. lief. 3. S. 269.) Sch.

Rochat (24) betont die Wichtigkeit fer epithelioiden Tuberkeln als häutigen Befund in Augen, welche eine sympathische Ophthalmic entweder erregt oder erlitten haben. Wie verschiedeac Autoren sah auch Verf. in der verdickten Aderhaut, im Ciliarkörper und in der Iris zahlreiche 
stark tingierte einkernige leukozyten und dazwischen zerstreut schwach gefirbte mit grossen ovalen Kernen; ron Verkäsung niemals eine Spur. Aus den in der Literatur nierlergelegten Fällen und aus den eigenen Beobachtungen geht hervor, dass man diese Veränderungen nur in den frül\%eitig enukleierten Augen findet und wenn die Bintzïndung eine sehr hösartige war. Er reiht dieselben dea infektiösen Granulomen mit unbekanntem lontagium an. Bei der nicht-sympathisierenden Cyklitis lıat man solche Veränderungen nie gesehen. - Wegen der Seltenheit der Ätiologie verdienen unter Verfassers Fiillen von sympathischer Ophthalmie besondere Erwähnung ein Fall nach Panophthalmie und Phthisis bulbi unl ein anderer nach killiveriit\%ung. Sch.

be Haas (12) hat nach verschiedenen Richtungen Versuche iiber die elektrischen Ströme im froschauge angestellt. \%ur br\%eugung ilerselben durch lichtrei\%e (Aktionsströine) braphte er das duge in einen Dunkelkasten, welcher :n einer Seite mit einem Schirme aus Seidenpapier versechen war; das letztere konnte durch das licht einer Lampe diffus beleuchtet werden, welches mittelst einer lionvexlinse auf das Seidenpapier gesammelt wurde. Bine Irisblende kurz vor der Konvexlinse ermöglichte es, die Beleuchtung des Seidenpapieres ab\%ustufen, olıne die (irösse der bestrahlılen Fläche zu ändern. Weil nun die beleuchteten 'l'eile des Schirmes den Lichtrei\% für das Auge bildeten, war dalurch erreicht. dass die Net\%laut immer ein gleich grosses Bild mit wechselncler Lichtintensitiit erhielt. Die Dauer der Belenchtung wurde reguliert durch Scheiben mit ausgeschnittenen Sektoren, welche kur\% ror der Lampe drehten. Ohne Beleuchtung war die Binrichtung \%ur Messung der Ruliestrïme geeignet. - Als bilektroden wurlen solche aus feinen Tondrähten, in $\mathrm{NaCl}$ getriinlit, am besten befunden. - Von dem Ruluestrome sei erwïhnt, lass Verf. lenselben un so stïrker fand, je näher der Lamina cribrosa die eive Blelitrode aufgesetzt war (am liebsten a $1 f^{\circ}$ der lamina) und je nïher der Hornhaut die andere. Wine negative Zone beim Iquator (Ilolmgren) wurde nur einmal konstatiert. Über die Aktionsströme sei mitgeteilt, dass dieselben nach momentaner Beleuchtung oft nicht weniger als $30^{\prime \prime}$ wïluten. lïin plötzliches Aufhören des Jichtreizes wirkt \%war viel schwiicher, aber immer im gleichen Sinne als der Lichtrei\% selber. - Wenn Ve:f. \%wei (jegenstinde beobachitet, welche rerschieden stark beleuchtet and ungleich lange sichtbar siucl, aber in dem Sinne, dass das Produkt aus Beplenchtungs. Intensitiit, und Sichtbakeits-1)aner für beide Gegenstincle stets lasselbe ist. nimmt er imuer \% wei gleichgrosse Gegenstiunde wahr, solange die SiclitbarkeitsJatuer $0,0 f^{\prime \prime}$ nicht iiberschreitet: beim froschauge aber folgte anf ein immer gleiches P'rorlukt ans Beleuchtungs-Intensitit und Reizdauer noch dieselbe photo-elektrische Reaktion, bis die letztere uuf $8^{\prime \prime}$ gestiegen war. bine solche lange Dauer gilt also für das Froschauge noch als Moment-Reiz; (las wird auch dadurch bestitigt, dass die photo-elektrische Reaktion dieselbe bleibt, wenn man eine gewisse Reizstairke entweder ununterbrochen oder in Intermissionen anwenclet. wenn nur die Dauer les Rei\%es $8^{\prime \prime}$ nicht übersteigt. Die Versuchsanordnung machte es möglich, den Einfluss der Adaptation fïr verschierlene Farben auf die Retinaströme \%u beurteilen während der farbigen Beleuchtung. So wie II aller mit einer successiren Methode gefunden hat, konnte Verf. mit 
seiner simulanen Methorle feststellen, dass die Arlaptation fiir eine beliebige Farbe alie Alitionsströme nach allen farbigen Reizen in gleichem Masse abschwicht. Er vermutet darum Farbenblindlieit ales Froschanges. - Die quantitative Beriehung zwischen Reizstiirke und Stromst.iirke ist bis jet\%t nur für ein selır kleines Gebiet aler wahrnehmbaren lichtintensitaiten untersucht worden durch Waller. Verf. hat die lintersuchungen ansgedehnt bis iiber die Hälfte dieses grossen Gebietes. Nennt man den schwiichsten wahrnehubaren Lichtreiz 1, dann wird der stairkste, für das Auge noch unschiilliche, dargestellt durch 10 13.)

In dieser untersuchten Hälfte wies die photo-elektrische Reaktion sehr bedeutende Abweichungen vom Fechnerschen Gesetze auf. Wenn man die lichtintensitiiten Iogarithmisch auf die Abscissen-Achse einteilt und als Ordinaten die Stromstiirken auftrïgt, fordert das genannte Geset\% fiir die Beziehung zwischen diesen beiden eine gerade linie, welche nach rechts aufwärts geht. Instatt dessen erhielt Verf. eine krumne Linie mit der Konvexität uach unten und rechts. Im noch nicht untersuchten (iebiete, bei sehr starken Iichtintensititen, wird, so meint Verf., die linie die Konvexitit nach oben wenden. - Verf. nimmt an, dass die photo-elektrische Realitionsstiirke in einem festen Verhiiltnisse \%u der Intensitiit der Gesichtswahrnehmung stehen muss; unter "lieser Vorausset\%ug gestatten uns die Frgebuisse dieser Intersuchungen einen Blick auf die Prozesse, aus welchen ile Wahrnehmungen mit dem Auge zusammengestellt sind.

s. ch.

Wie anclere Lntersucher, fand auch van Halten (11) bei Conjunlitivitis phlyctaenulosa den Staphylococcus aureus sehr oft in ïppigen Kulturen und immer in viel grösseren Quantitïten als auf der gesunden Bindelıaut. Es könnte sein, dass die krankhafte Hyperimie des Auges diesen Staphylococcus $\approx$ besserem Gedeilıen Gelegenheit gäbe; dats wird aher dalurch widerlegt, dass Verf. ihn bei anderen hyperämischen $\mathrm{Zu}$ stiinclen (durch Corpus alienum corneae, bei lritis u. s. w.) nu! in geringer Menge rorfand. Vielmehr erblickt Verf. in diesem Staphylococcus die Vrsache der phlyktiinulösen lintæündung. Derselbe stamınt, wie fast mit Gewissheit behauptet werden kann, von ilenjenigen Staphỵlokokken her, welche der normulen Bindehant so gut wie immer anhaften. Warum werlen dieselben plötzlich pathogen? Daran ist die Skrophulose schuld, len॥ wenn Verf. Kaninchen tuberkulös gemacht hatte, reagierte deren IIornhaut viel heftiger nuf eine StaphylokokienInfektion, als das bei normalen 'l'ieren der liall ist. Sch.

l) (Bruind (1) schlägt vor, allen Neugeborenen 2 pC't. Nitr. arov. rin\%utrüuteln. Scli.

Bourin (t) berichtet ïber den Kranken mit Pemphigus conjunctivate, welchen er ror anderthalb Jahren vorgestellt hat (vergl. ien Bericht fuir das 1. Semester 1902). Let\%terer hist sich bedeutend gebessert, es ist keine Xerosis, sondern nur ein kleines laterales Symblepharon aufgetreten. Sch.

lilias (7) gibt eine Übersicht des Pupillenspieles im krankhaften und gesunden Zustandle. Die Darstellung weicht darin ron der gewöhnlichen $u b$, dass Verf. den $M$. dilat:itor pupillate nicht in die Iris verlegt, sonılern in den Ziliarkörper, und dass er sich den Ziliarmuskel bei Myopie in einem kontralierten Zustande denkt. sich. 
Ilazewinkel (13) gibt eine Illustration zu dem Geset\%, lass Augen, welche lisen enthalten, früher orler spaiter \%ugrunde gehen. Die llitteilung betrifft ein Auge, welches vor 11 Jahren durch einen Eisensplitter verwundet wurle, j) Jahre spaiter noch $S .=1 / 8$ aufwies, bei einer leicht roriibergehenden lritis, und jet\%t ler Sirlerosis anheimgefallen ist: rotbraune Verfäbung der atrophischen, reaktionslosen Iris, braune, punktförmige Trübungen in den tieferen Hornhautschichten, Imaurose, also wahrscheinlich Atrophie der nicht mehr sichtharen Net\%haut und Iridorlonesis als erstes Zeichen rler Schrumpfung. Scli.

Straub (31) betont, dass man wedes die Amblyopia ex anopsia, noch die Anopsia ex amblyopia verneinen kann. Aber auch keine dieser beiclen Hypothesen erklärt alle lirscheinungen: öfters hat Verf. Amblyopie olıne Anopsie gefunden, nämlich , lie einseitigen Amblyopien olune Strabismus, welchen man nicht selten bei den Militiirpriifungen begregnet (vergl. Straub, Archiv f. Augenlieilk. 1896). Ungekehrt kann die Amblyopie nicht immer primär sein, weil das Auge sich oft bessert, wenn man ihm Gelegenheit \%ur Übung gibt, \%. B. durch Schieloperationen. Verf. teilt dariiber einen sprechenden Fall mit. - Nun hat Str. bei der einseitigen Amblyopie fast immer Hypermetropie gefunden. Jir erinnert daran, dass viele Hypermetropen, auch solche mit \% wei guten Augen unal binokularem Sehen, ein "oeil directeu" besitzen (Tscherning). Diese Tatsache, dass ron \% wei normalen Augen das eine viel wichtiger sein kaun, sowohl was die Erregung der Netzhaut wie die Innervation der Muskeln betrifft, betrachtet Verf. als die primäre Abweichung. Dieselbe kinn sowohl \%u Strabismus (mit oder oline Amblyopie; wie «u Amblyopic (mit oder ohne Strabismus) fiilıren. line weitere Frage bleibt nocl often: (ob die Hypermetropie - die Refraktion der säuglingsaugen - besteher bleibt, weil das Binokularsehen schwach angelegt ist, oler aber, ob das Binokularsehen schwach wirl, weil die Hypermetropie persistiert.

Sch.

1) yckmeester (6) beschreibt ien folgenlen ophthalmoskopischen Befund: In einem gesunden hypermetropischen duge sall er in dem unteren äusseren Quadranten der Sehnervenpapille eine seichte Aushöhlung von blallschwar\%er farbe mit olivgrünem Glan\%e. liine Retinal-Vene verlief bis \%um Ranle dieser gefïirbten Stelle und wurle daselbst unsichtbar. Verf. betrachtet die Anomalie als eine liongenitale Anhïufung ron Net\%hautpigment.

Sch.

binen anderen Befund hat van (ieuns (10) ver\%eichnet: In deu klaren Glaskörper eines astigrmatischen Auges lrang eine arterielle Gefitisschlinge 6 I). Weit hinein. Bei der einmaligen Beobachtung, welche bei dem ängstlichen Kindle nicht gan\% sicher war, schien die Schlinge aus dor A. centralis retinate herormukommen und sich in die $A$. temp. infer. fort\%usetzen.

Sch.

In einem geringen Vordringen der Arterien und Venen in den (ilaskiorper erblickt faber (8) ein Zeichen, dass die Papille selbst hervortritt. Er nennt diese Eigentumliclıkeit "Papilla conica" und hat dieselbe oftmals gefunden in gesunden Augen und auch bei verschiedenen hrankheiten, ja selbst bei Atrophie les Sehnerven. lis geht aus der Mitteilung nicht deutlich hervor, ob wirklich eine prominierende Papille 
gesehen wird oder ob dies nur ans dem Hervortreten der Gefässe geschlossen wird.

Sch.

Über die Vena optico-ciliaris, welche van Gieuns (9) sich waihrend einer Papillitis entwickeln sah (s. rorigen Bericht), teilt er weiter mit, dass das Gefäss sich gänzlich \%urückgebildet hat, während die lint.zündung in eine neurotische Atrophie der Papille iibergegangen ist. S. ist jetrt mehr als $\% / 10$, das Gesichtsfeld auf beiden Augen nasal eingeschräinkt.

sch.

Schoute (28) nahm einen linophthalmus tramaticus wahr: Beinr Üfinen einer Blechbuichse schlug der Hofmeister eines Schiffes sich selbst mit ler Faust gegen den oberen Orbitalrand. Die verletzte Stelle war mit. Blut unterlaufen und das vorher normal stehende Auge in die Orbita hineingesunken. Sch.

Noyon (20) berichtet folgendes: liner Krankenwärterin war ein Carcinom der Parotis fortgenommen worden mit Aufopferung de: $\mathrm{N}$. facialis und der A. carotis externa. Wine kleine krat\%wunde auf der Hornhaut der gelähmten Seite führte später eine langdauernde Keratitis herbei; obwohl ausser dem fiacialis auch der Abrlucens und Trigeminus gelähmt gefunden wurder, war das Auge der Sit\% lieftiger Schmer\%en, weil nïmlich Patientin an Neuralgien \%u leiden anfing, welche sie in das Auge verlegte. Die suggestive Wirkung ciner optischen Iridektomie auf dem bereits sehr abgeschwiichten Auge vermochte die Neuralgien nicht \%um Verschwinden $\%$ bringen, sie wurden bloss nach dem Hinterkopfe verlegt und damn eine Zeit lang mit Chinin bezwngen. Bald stellten sic sich aber wieder halbseitig im liopfe ein; das Auge, von einem Pannus crassus äberzogen und nahezu blind, wurde aut Wunsch der Patientin entfernt: die Neuralgien schwanden nicht. Nach dieser letzten Operation wurde Patientin ron Schwindelanfillen getrofien. Is nahm oline andere objelitive Erscheinungen als die Aufhebung der Pupillenreaktion auch die Sehkraft des übriggebliebenen Auges allmählich ab, bis es schliesslich erblindete. Auch darn hielten die Neuralgien und Schwinclelanfälle unverändert an. Verf. nimmt als Ursache der Blindheit sowie der subjektiven Frscheinungen ein Reridiv des Carcinoms innerhalb der Schädelhöhle an mit Blutungen ins Chiasma oder N. opticus. Verzeichnet muss werden, dass Pat. vicht an Hysterie litt und dass weder in der Orbita oder Nase noch in den Nebenhühlen etwas Krankhaftes zu entrlecken war. Merkwürdig ist, lass nach liingerer Daner der Blimblheit noch inmer nicht mit Sicherheit eine: Atrophie der Sehmervenscheibe festgestellt we:den konnte. sch.

Pecters (2I) beschreibt dis Auftreten ron akuten Glaukomanfilllon nach einem Schrecken. Dieses ätiologische Noment reizt den Srm. pathikus, verursacht dadurch einen Gefässkrampf der kleinen Venen, also eine Staunng. welche zur Erhöhung der Spannung des Auges fïhren soll.

sth.

Die Krankengeschichte, welche Ruys (2j) mitteilt, ist die eines älteren Mannes, der nach einer belleutenden Anstrengung bewusstlos gefunclen wurde. Der Getroffene schiclte stark konvergierend und die Reflexe des ganzen Körpers, mit Ausnahme derjenigen der Pupille, waren aufgehoben. Weun der Mann erwachte, klagte er über Kopfschmer\% und Doppeltsehen: alle Muskeln waren ihm steif. Es handelte 
sich, Was die Augen betriftt, 1 m eine akute doppelseitige Abducenslaihmung; ausserdem wies jedes Auge eine Stauungspapille auf mit einer "rrossen Netzhautblutung. In wenigen Monsten wurde der Kranke geheilt: lie Augensymptome verschwanclen am spaitesten. Wahrscheinlich hatte eine Blutung stattgefunden in der Gegend les vierten Hirnrentrikels. Scli.

Stark (30) hat eine Jarstellung der lindehauterkrankungen für Laien gegeben, Pinklioff (22) eine selır fassliche und doch kurze Beschreibung der Laientuirsorge gegen Augenerkrankungen, eine freie l'marbeitung eines gleichen dufsatzes in der "Deutschen Praxis".

Bijlsma (2) hot einen Jeitfaden für ḱrankenwiirter geschrieben.

X. X., ein Laie (1S), hat durch Verwechslung ron griinem und graucm Staar seine Arbeit selbst verurteilt.

Sch.

Straub (32) empfiehlt, die lanze für alle fälle mit Taylors geknicliten Schmalmesser \% vertauschen. So wie das Gräfesche Messer das Beersche verdrängt hat, weil nur das erstere frei zu manöverieren gestattet, so muss aus denselben Griuclen das 'Taylorsche Vesser die breiten Janzen verdriingen.

Sch.

Salomonson ( 26 ) berichtet über \%wei fialle ron Ulums rodens des linterlicles, gelıeilt durch Röntgenstrahlen; einer dieser Patienten ist seit 8 Monaten rezidivfrei, der andere noch in Behandlung. Sch.

lechner (16) hat einen fall von angeborener Wortblindlieit bekannt gegeben, welcher in allen Einzelleiten den anderen gleicht, auf welche in letzterer \%eit die Aufmerksamkeit gelenkt worden ist. Er betraf einen Jungen mit gutem Verstand und ganz normalen Augen; allein es war ihm nicht möglich, lesen zu lernen. Er kennt nur ver. einzelte Buchstaben. Er lıört gut, kann vorgesagte Wörter fehlerfiei nachsprechen, ohne Aphasie noch Paraphasie. Spontan schreiben kann er nur seinen Namen; beim liopieren macht sich l'aragraphie bemerkbar. Wie in den anderen fiallen auch rermerkt wurde, kann er die Viftern lesen und schreiben ohne jedwede Beschwerde.

sich.

\section{Gesellschaftsbericht.}

\section{Berliner Ophthalmologische Gesellschaft.}

Sitzung rom 16. Februar 1905.

Vorsitzender: Herr v. Nichel.

Schrittührer: Herr Wertheim.

Herr llirschberg: Kranken-Vorstellung. Der Patient erlitt :m 31. NIl. 1904 eine Verlet\%ung nit Hypopyonbildung und Hämorrhagie Wer unteren Net\%autpartie: bei Anwendung des Handmagneten buckelte sich die Iris ror und es reigte sich ein Eisensplitter, der lann mittels schnittes und Magneten leicht entfernt wurde. Jir ist ca. $13 \mathrm{~mm}$ lang, 